\title{
AIRBORNE MULTIDIMENSIONAL INTEGRATED REMOTE SENSING SYSTEM
}

\author{
Weiming Xu*, Jianyu Wang, Rong Shu, Zhiping He, Yanhua Ma \\ Shanghai Institute of Technical Physics, CAS, 500 Yutian Road, Shanghai 200083, P. R. of China - \\ xuwm@mail.sitp.ac.cn
}

Commission VI, ICWG V/I

KEY WORDS: Imaging spectrometer, Three-line scanner, Laser ranger, Position \& orientation subsystem

\begin{abstract}
:
In this paper, we present a kind of airborne multidimensional integrated remote sensing system that consists of an imaging spectrometer, a three-line scanner, a laser ranger, a position \& orientation subsystem and a stabilizer PAV30. The imaging spectrometer is composed of two sets of identical push-broom high spectral imager with a field of view of $22^{\circ}$, which provides a field of view of $42^{\circ}$. The spectral range of the imaging spectrometer is from $450 \mathrm{~nm}$ to $900 \mathrm{~nm}$, and its spectral resolution is $5 \mathrm{~nm}$. The three-line scanner is composed of two pieces of panchromatic CCD and a RGB CCD with $20^{\circ}$ stereo angle and $10 \mathrm{~cm}$ GSD(Ground Sample Distance) at $1000 \mathrm{~m}$ flying height. The laser ranger can provide height data of three points every other four scanning lines of spectral imagers and those three points are calibrated to match the corresponding pixels of the spectral imagers. The post-processing attitude accuracy of POS/AV 510 used as the position \& orientation subsystem, which is the aerial special exterior parameters measuring product of Canadian Applanix Corporation, is $0.005^{\circ}$ combined with base station GPS data. The airborne multidimensional integrated remote sensing system was implemented successfully, performed the first flying experiment on April, 2005, and obtained satisfying data.
\end{abstract}

\section{INTRODUCTION}

Both the spatial and spectral resolutions are very important specifications for remote sensing systems. The former determines geometrical resolution power of systems; the latter determines the power of target recognizing. In the past few decades, the spatial and spectral resolutions of airborne remote sensing systems have been improved greatly. The spatial resolution reached $0.1 \mathrm{~m}$ at $1000 \mathrm{~m}$ flying height, and the spectral resolution up to $1 \mathrm{~nm}$. However, it is still very difficult to obtain good images with both high spatial and spectral resolution simultaneously, which can be registered easily, because of relative restriction of these two specifications. To fulfill the requirements, we designed this multidimensional integrated remote sensing system that can provide high spatial and spectral data synchronously. This system was supported by high-tech research and development program of China, which can survey and produce 1:2000 to 1:5000 thematic map and suitable for the regions of city layout, resource investigation, environment inspection, similar applications and so on.

\section{SYSTEM DESCRIPTION}

\subsection{System configuration}

The system consisted of multidimensional information acquisition subsystem, multidimensional information processing subsystem, performance measurements and systematic calibration subsystem. The information acquisition subsystem includes: a three-line scanner with high spatial resolution power, an imaging spectrometer with high spectral resolution power, a laser ranger, a stabilized platform and a set of attitude \& position measuring device. The multidimensional information processing subsystem includes: data preprocessing module, high spatial data processing module, high spectral data processing module, and data merge processing module. The performance measurements and systematic calibration subsystem includes: comprehensive performance measurement module in the lab, spectral and radiometric calibration in the lab and field. All the above are shown in figure 1 and 2 .

As shown in figure 2, the system can be used as one set of device, also can be divided into two parts: high spatial part and high spectral part, which is flexible for different applications. When used as a whole, the system can be controlled by a computer; consequently it needs only one person to operate this instrument. And a basic external clock was divided into different frequencies to synchronize all sensors: $600 \mathrm{~Hz}$ for three-line scanner, $50 \mathrm{~Hz}$ for imaging spectrometer, $10 \mathrm{~Hz}$ for laser ranger, at the same time all the working frequencies were copied to POS/AV 510, which could record attitude and position information at the rising or falling edge of the ingoing pulse according to the customized settings.

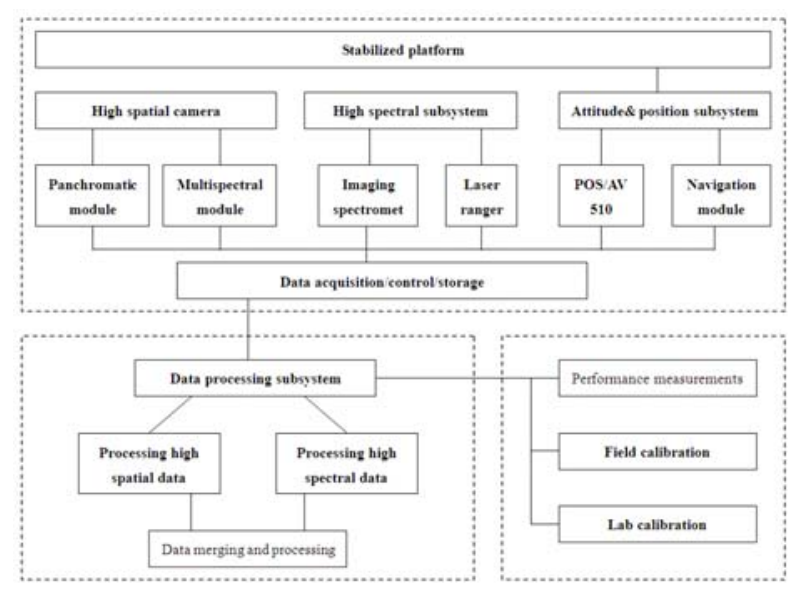

Figure 1. Functional diagram of system 


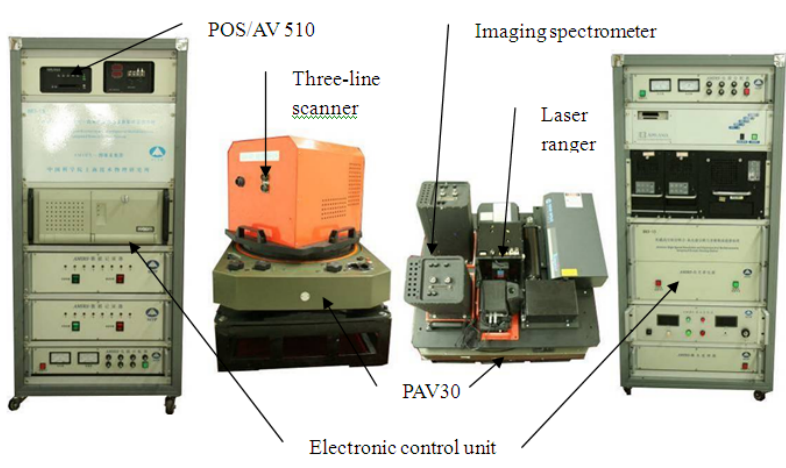

Figure 2. System overview

\subsection{Technical specifications}

The following list is technical specifications:

- Mapping scale: 1:2000 - 1:50000;

- FOV(Field of View): $42^{\circ}$;

- Spatial pixels:

- 10200 pixels for three-line scanner

- 1278 pixels for imaging spectrometer

- 3 points each line for laser ranger

- Spectral range:

- 465-680nm for panchromatic image, 1 band

- $\quad 610-660 \mathrm{~nm}, 535-585 \mathrm{~nm}, 430-490 \mathrm{~nm}$ for multispectral image, 3 bands

- 450-900nm for hyperspectral image, 244 bands, spectral resolution better than $5 \mathrm{~nm}$

- Instantaneous FOV:

- $0.1 \mathrm{mrad}$ for panchromatic and multispectral image

- 0.6 (across track)*1.2mrad(along track) for hyperspectral image;

- Laser distance resolution: $7.5 \mathrm{~cm}$;

- Scanning rate:

- $300 \mathrm{~Hz} / 600 \mathrm{~Hz}$ for three-line scanner

- $50 \mathrm{~Hz}$ for image spectrometer

- $10 \mathrm{~Hz}$ for laser range;

- Radiation resolution: 12 bits;

- Stereo angles: $-21^{\circ}$ backward, $0^{\circ}$ nadir, $20^{\circ}$ forward;

- Accuracy of attitude and position measurement

- Real-time: position $4-6 \mathrm{~m}$, roll $/$ pitch $0.008^{\circ}$, heading $0.07^{\circ}$;

- Post-processing: 5-30cm, roll/pitch $0.005^{\circ}$, heading $0.008^{\circ}$;

- Stabilized platform:

- Stabilized range in roll/pitch: $\pm 5^{\circ}$;

- Stabilized range in drift: $\pm 30^{\circ}$;

- Typical residual angular motion: $<0.3 \%$;

- Typical residual deviation from vertical (nadir): $\pm 0.5^{\circ}$.

\section{MODULAR DESIGN OF SUBSYSTEMS}

As shown in figure 2, our system employed modular design completely, wherefore every module can be implemented respectively, and also can be used separately.

\subsection{Design of wide angular FOV imaging spectrometer}

Generally, pushbroom imaging spectrometer has a field of view less than $30^{\circ}$ due to pixel constraints of linear array CCDs. This limited its working efficiency and increased working cost. For higher efficiency, we combined two imaging spectrometer with
FOV $22^{\circ}$ to achieve a wide angular FOV $42^{\circ}$. They were overlapped $2^{\circ} \mathrm{FOV}$ and fixed on a whole plate, as shown in figure 4. The dispersing optical construction employed prism-grating-prism spectrograph, shown in figure 3, which is compact and has a high optical efficiency. All CCDs were kept in a constant temperature via a cooling system to increase the signal to noise ratio.

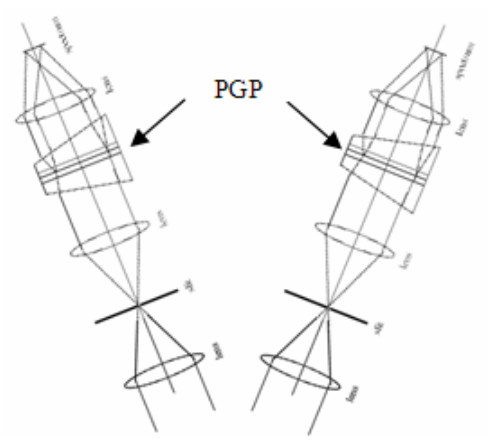

Figure 3. Schematic diagram of optical splicing

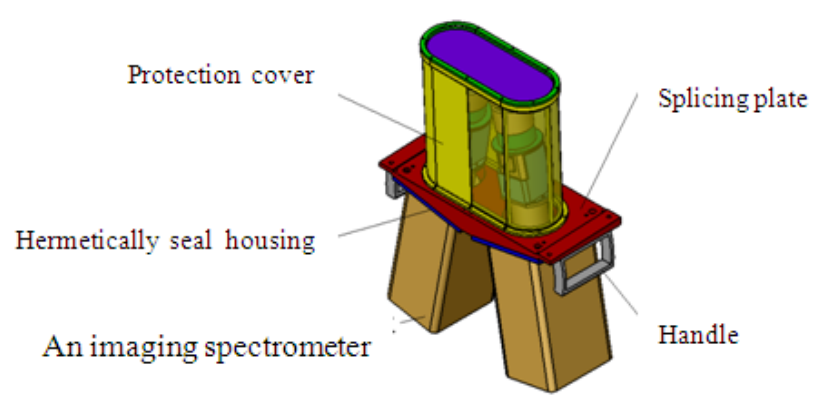

Figure 4. Wide angular FOV imaging spectrometer

\subsection{Design of three-line scanner}

The three-line scanner was designed according to the principle of linear stereo imaging: three CCDs image same scene respectively by forward view, nadir view and backward view approximately at the same time. The designed stereo angle was $20^{\circ}$, thus we can calculate and obtain three dimensional coordinates of every pixel theoretically according to photogrammetric principle. In the focal plane, we set two panchromatic CCDs, one RGB CCD that contains red channel, green channel and blue channel, namely five channels. RGB $\mathrm{CCD}$ was centered in the focal plane, as shown in figure 5. Two panchromatic were moving forward and backward by about the same distance, which depended on the ratio of base line and flight altitude. In this design, the ratio is 0.73 because of the restriction of flat field area of focal plane.

An objective was designed for all CCDs, whose focal length is $75 \mathrm{~mm}$. The CCDs is the products of Kodak Corporation. The pixel size is $7 \mu \mathrm{m}$, whereby an instantaneous field of view of $0.1 \mathrm{mrad}$ was achieved. 


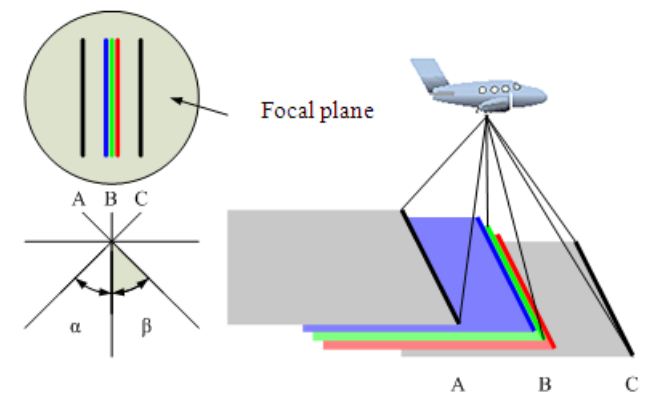

Figure 5. Construction of focal plane and principle of stereo imaging

\subsection{Design of laser ranger}

Laser module gave three footprints every other four hyperspectral image lines, shown in figure 6. The laser footprints were registered strictly with the hyperspectral image pixels, which was fulfilled by opto-mechanical design as a whole consideration and calibration in the lab.
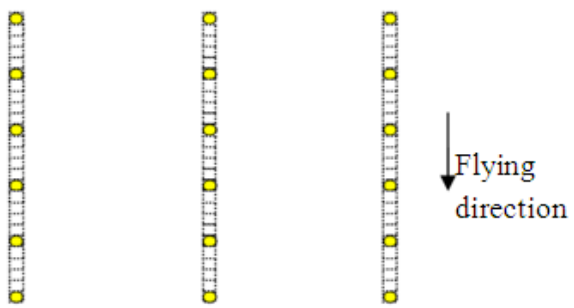

Figure 6. Registration of laser footprints and hyperspectral pixels

Laser module didn't employ any scan components, but adopted two beam splitters to obtain three desired points from a laser beam, shown in figure 7 . The original laser beam was divided into three equivalent portions by energy. The right and left beam were directed at $\pm 11^{\circ}$ with respect to central vertical beam.

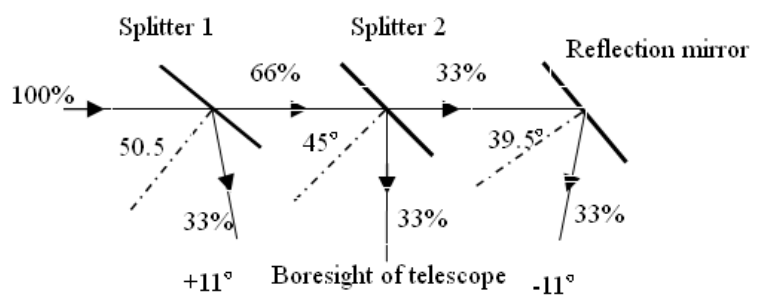

Figure 7. Drawing of laser beam splitting

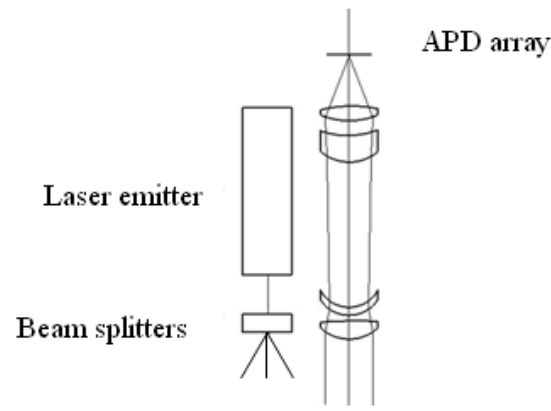

Figure 8. Optical construction of emitting and receiving
The energy of laser emitter in our system is less than $100 \mathrm{~mJ}$, the divergence angle is better than $3 \mathrm{mrad}$, the repetition frequency is $20 \mathrm{~Hz}$, and the central spectrum $1064 \mu \mathrm{m}$. The optical aperture of receiving optics is $60 \mathrm{~mm}$, and IFOV $4 \mathrm{mrad}$, shown in figure 8. The left part emits laser beam, and the right part receives reflection beam, then is converted into electronic signal through APD (Avalanche Photodiode). A precise time interval measuring board was deployed to arbitrate and calculate the time interval between emitted pulse and received pulse, which ensured the distance resolution of $7.5 \mathrm{~cm}$.

\subsection{Stabilized platform and attitude \& position measurement subsystem}

It is pretty common to deploy stabilized platform and attitude \& position device in photogrammetric and remote sensing system nowadays. In virtually, this is not the best method, perhaps a high enough precise three dimensional stabilized platform is better in theory, however the former is still more reasonable and flexible on account of current situation of research, development and cost.

In our system, the stabilized platform, PAV30 of Leica Corporation, was employed to stabilize imaging sensors. Though the accuracy of PAV30, $\pm 5^{\circ}$, is not very high, it is enough for general airborne application and basically ensures that there are no big gaps during and between flight strips, which is very important for a successful application.

Moreover, we installed a start-of-the-art GPS/INS system, POS AV/510, developed by Applanix Corporation. POS AV/510 device can provide real-time and post-processing delivery of attitude and position with excellent accuracy and precision. It operates using combined IMU (Inertial Measurement Unit) and GPS (Global Positioning System) receiver. The position information from the GPS system and the 3-axis attitude and position data from IMU are combined in a tightly Kalman filter to provide $\mathrm{x}, \mathrm{y}$ and $\mathrm{z}$ position values as well as roll, pitch and true heading. The stated accuracy is $5-30 \mathrm{~cm}$ in position, 18 " in roll and pitch, 28.8" in true heading when operated with differential GPS and made a post-process.

All sensors, including one of POS AV/510, are bolted directly to platform frame, and must be prohibited from any relative motions among them.

\subsection{Data acquisition and storage subsystem}

In this system, we employed a master control computer to operate all sensors, as shown in figure 9. The connection interface between the master computer and sensors is local network. The operator interface controls work mode and status of all sensors, and also receives some image data to monitor through net cables. After commencing to acquiring data, all sensor modules sent an event pulse, which marked the exposure moment of every scan line, into POS AV/510.

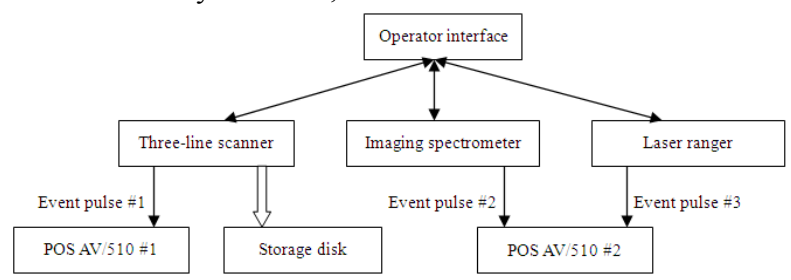

Figure 9. Working flow of data acquisition and storage 
disk for 4 hours flying. However, the three-line scanner data can not be stored like this. The data rate of three-line scanner is $35 \mathrm{MByte} / \mathrm{s}$, and the data amount will be up to $500 \mathrm{~GB}$ for 4 hours flying. On account of this, we decided to employ advanced SCSI programming interface to transfer data, which skips windows' file system to store data into the RAID disks directly. The capacity of extending RAID disks achieves 560GB, and we deployed two sets of the storage device, hence it can fulfil the requirements of 4 hours continuous work.

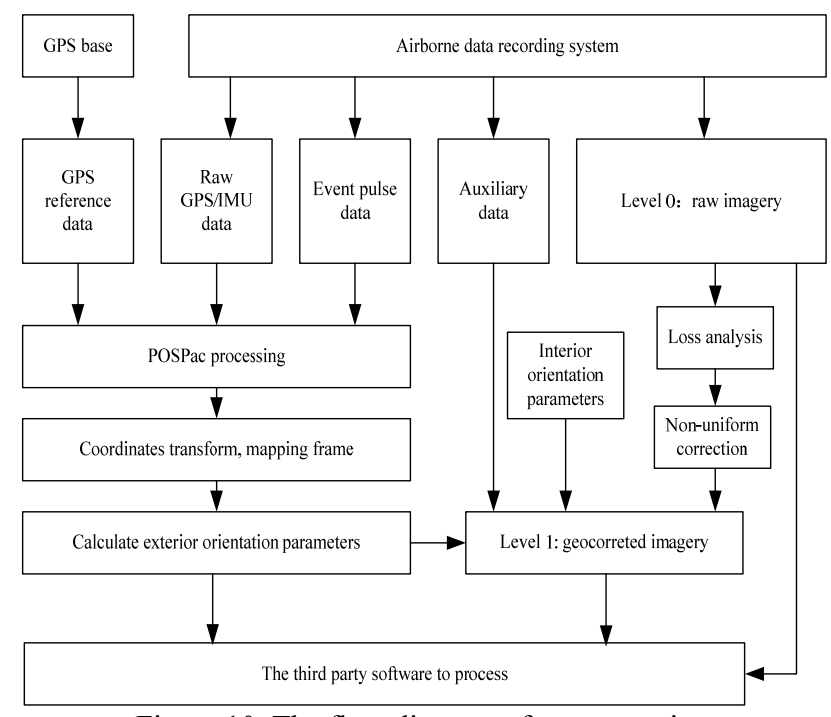

Figure 10. The flow diagram of geocorrection
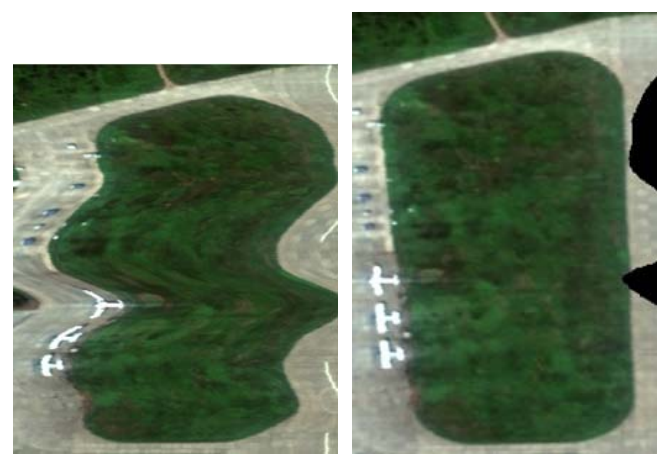

Figure 11. Raw and geocorrected hyperspectral imagery
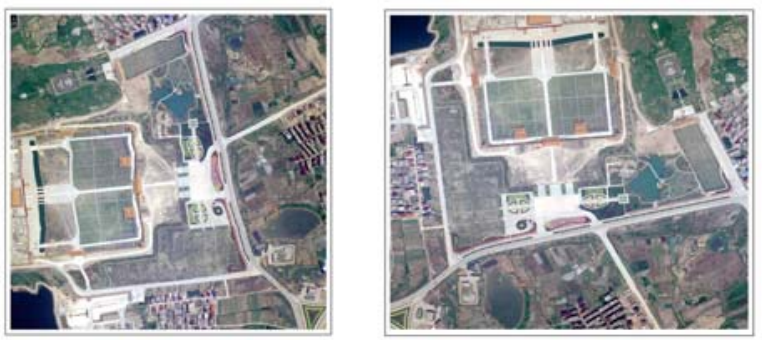

Figure 12. Raw and geocorrected high spatial imagery

\subsection{Digital processing subsystem}

After a successful flight, we obtained high spatial data, hyperspectral data, laser ranging data, attitude and position data, and all other required auxiliary data. Therefore, the data processing subsystem also contains following modules: high spatial program module, hyperspectral program module, laser program module and attitude \& position program module, as well as a program module to merge all sensors and extract the required information. Attitude \& position program module is POSPac software developed by Applanix Corporation. Figure 10 indicates the working flow to process the high spatial data.

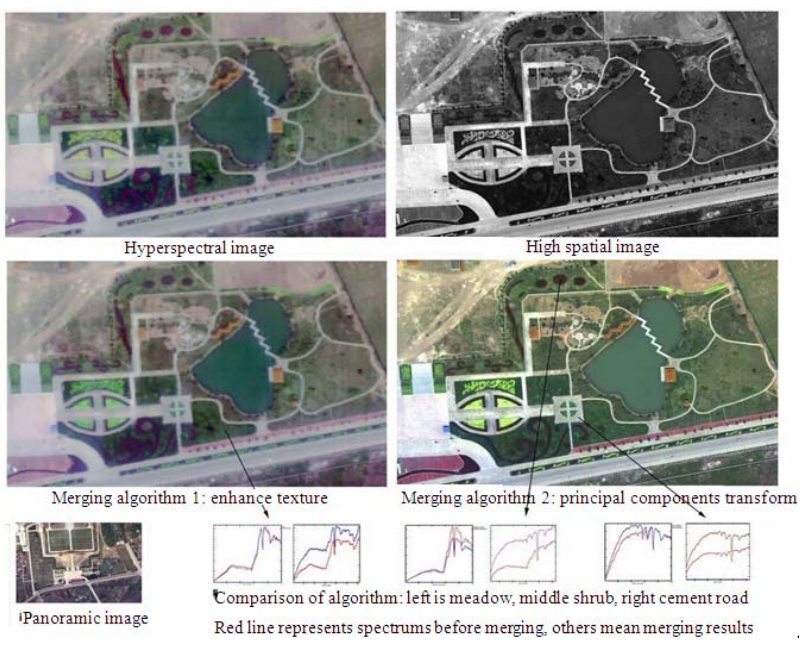

Figure 13. Diagram of imagery merging. The top left is hyperspectral images, the top right high spatial images, the middle left merging results through filter algorithm in frequency domain, the middle right another merging results through principle components transformation, at the bottom isthe comparison of spectrum curve before and after merging.

\section{SUMMARY AND CONCLUSIONS}

Airborne multidimensional integrated remote sensing system was finished on 2005. This system can provide high ground resolution panchromatic and multispectral imagery and hyperspectral imagery as well as laser ranging data simultaneously in one flight. The imaging operation is controlled by appropriate software implemented in the master computer connected to all sensors via net cables. The distortions caused by the fight dynamics of the aircraft are rectified using the high precise information extracted from the position and attitude measurement device developed by Applanix Corporation. The standard data format is provided for users' convenience to apply the commercial processing software.

\section{ACKNOWLEDGEMENTS}

Great thanks to my colleagues who participated and made great contributions in this project, including Li Zhang, Yuwei Chen, Peixin Hu, Binla Zhang and Qimin Lu et al. Special thanks are expressed to Mrs. Kangmei Fang for her contributions to optical calibrations in this system.

\section{REFERENCES}

Applanix Corporation, POS AV/510 Specifications, http://www.applanix.com (accessed Feb. 12, 2008).

LH systems technical reference manual, "LH systems PAV30-Gyro-stabilized camera mount”, 2001.

Rainer Sandau, Bernhard Braunecker, Hans Driescher, Andreas Eckardt et.al, "Design principle of the LH systems ADS40 airborne digital sensor", IAPRS, Vol XXXIII, Amsterdam, 2000.

Technical manuals (interior communication). 